\title{
Alain CAMPBELL \\ Impédance d'une plaque élastique reliée en trois points à un support rigide vibrant
}

Annales de la faculté des sciences de Toulouse $5^{e}$ série, tome 10 , no 2 (1989), p. 199-213

<http://www.numdam.org/item?id=AFST_1989_5_10_2_199_0>

(C) Université Paul Sabatier, 1989, tous droits réservés.

L'accès aux archives de la revue «Annales de la faculté des sciences de Toulouse » (http://picard.ups-tlse.fr/ annales/) implique l'accord avec les conditions générales d'utilisation (http://www.numdam.org/conditions). Toute utilisation commerciale ou impression systématique est constitutive d'une infraction pénale. Toute copie ou impression de ce fichier doit contenir la présente mention de copyright.

\section{Numdam}

Article numérisé dans le cadre du programme

Numérisation de documents anciens mathématiques

http://www.numdam.org/ 


\title{
Impédance d'une plaque élastique reliée en trois points à un support rigide vibrant
}

\author{
Alain Campbell ${ }^{(1)}(2)$
}

RESUME. - Dans cet article on rappelle la notion de matrice d'impédance d'une plaque élastique reliée à un support rigide suivant un ensemble $\Sigma$ et on montre comment on peut calculer ces matrices si $\Sigma$ est de mesure strictement positive. Ensuite, par passage à la limite, on définit l'impédance dans le cas où $\Sigma$ est constitué de trois points non alignés.

\begin{abstract}
In this paper we explain the notion of impedance matrix in the case of an elastic plate which is fixed to a vibrating rigid support along a domain $\Sigma$ and we show how we can calculate these matrices if the measure of $\Sigma$ is strictly positive. Then, by using limits, we define the impedance when $\Sigma$ is contituted of three non collinear points.
\end{abstract}

\section{Introduction}

Dans l'étude du comportement dynamique de satellites on s'intéresse aux efforts exercés par les grands éléments flexibles, tels que les antennes ou les panneaux solaires, sur les éléments rigides auxquels ils sont fixés. Pour calculer ces efforts on définit la notion de matrice d'impédance [cf. 1]. Dans le cas de structures élastiques reliées à un bras rigide par une soudure de mesure strictement positive, des résultats explicites, obtenus par des méthodes analytiques, ont été donnés par exemple par KelKel [cf. 2] dans le cas de plaques circulaires ou rectangulaires. Des méthodes variationnelles fournissent aussi des procédés de calcul de matrices d'impédance y compris dans le cas de structure tri-dimensionnelles [cf. 3], et peuvent être exploitées numériquement par éléments finis.

(1) Laboratoire d'Acoustique et Mécanique, Groupe de "Modélisation et Calcul" UA 868

C.N.R.S., Université Pierre et Marie Curie; Tour 66, 4, place Jussieu 75230 Paris

(2) Laboratoire de Mécanique, Université de Caen 14032 Caen 


\section{A. Campbell}

L'objet de ce travail est de préciser le comportement limite de la matrice d'impédance d'une plaque élastique soudée à un bras rigide quand la mesure du support de cette soudure tend vers zéro. On étudiera particulièrement le cas où la plaque est fixée au support rigide en trois points non alignés.

Dans la première partie, on rappellera brièvement la notion de matrice d'impédance d'une structure élastique reliée à un support rigide et on montrera comment l'étude de problèmes variationnels permet d'obtenir une forme générale de ces matrices dans le cas d'une soudure de mesure positive (par exemple suivant un ou plusieurs disques de rayon $\epsilon$ ). On sera conduit à plusieurs théorèmes d'existence et d'unicité des solutions de ces problèmes variationnels.

La deuxième partie montrera que la plupart des théorèmes précédents restent valables si la fixation rigide se fait en trois points non alignés. La matrice d'impédance pourra alors être définie comme limite quand $\epsilon$ tend vers zéro des matrices de la première partie.

\section{Première partie : Notion d'impédance, existence et calcul}

\section{II.1. - Impédance d'une plaque élastique reliée à un bras rigide}

Soit $\mathcal{P}$ une plaque mince homogène isotrope d'épaisseur $h$ et de masse volumique $\rho$. On suppose que son comportement en flexion suit les lois de Love Kirchhoff. Cette plaque est reliée à un barreau rigide $\mathcal{R}$ par une soudure $\Sigma$ de mesure positive, par exemple un disque centré en un point $A$ de la plaque et de rayon $\epsilon$.

Le contour de la plaque $\mathcal{P}, \Gamma_{1}$ est suffisamment régulier, par exemple $\mathcal{C}_{1}$ par morceaux. On le suppose libre d'efforts. On note $A x_{1} x_{2}$ le plan de la plaque et $A x_{3}$ la direction orthogonale. Le mouvement de vibration rigide du barreau est défini par une translation

$$
U_{o} e^{i \omega t} \vec{x}_{3}
$$

et deux rotations

$$
\Theta_{1} e^{i \omega t} \text { et } \Theta_{2} e^{i \omega t}
$$

autour de $\overrightarrow{x_{1}}$ et $\overrightarrow{x_{2}}$ respectivement (cf. figure 1 ).

Les éléments de réductions en $A$ du torseur résultant des actions de $\mathcal{P}$ sur $\mathcal{R}$ sont une résultante

$$
\begin{gathered}
R_{o} e^{i \omega t} \vec{x}_{3} \\
-200-
\end{gathered}
$$




\section{Impédance d'une plaque élastique}

et un moment en $A$ :

$$
\left(M_{1} \vec{x}_{1}+M_{2} \vec{x}_{2}\right) e^{i \omega t} .
$$

La matrice d'impédance $\mathcal{I}$ est alors la matrice de l'application linéaire de $\mathrm{R}^{3}$ dans lui-même qui à $\left(U_{0}, \Theta_{1}, \Theta_{2}\right)$, associe $\left(R_{0}, M_{1}, M_{2}\right)$.

On note

$$
\mathcal{I}=\left[\begin{array}{lll}
I_{00} & I_{01} & I_{02} \\
I_{10} & I_{11} & I_{12} \\
I_{20} & I_{21} & I_{22}
\end{array}\right]
$$

Cette matrice dépend de la pulsation $\omega$ du mouvement de $\mathcal{R}$, du point $A$ et de la soudure $\Sigma$, donc de $\epsilon$. C'est cette relation $\mathcal{I}(\epsilon)$ qu'on se propose d'étudier ici en particulier quand $\epsilon$ tend vers zéro.

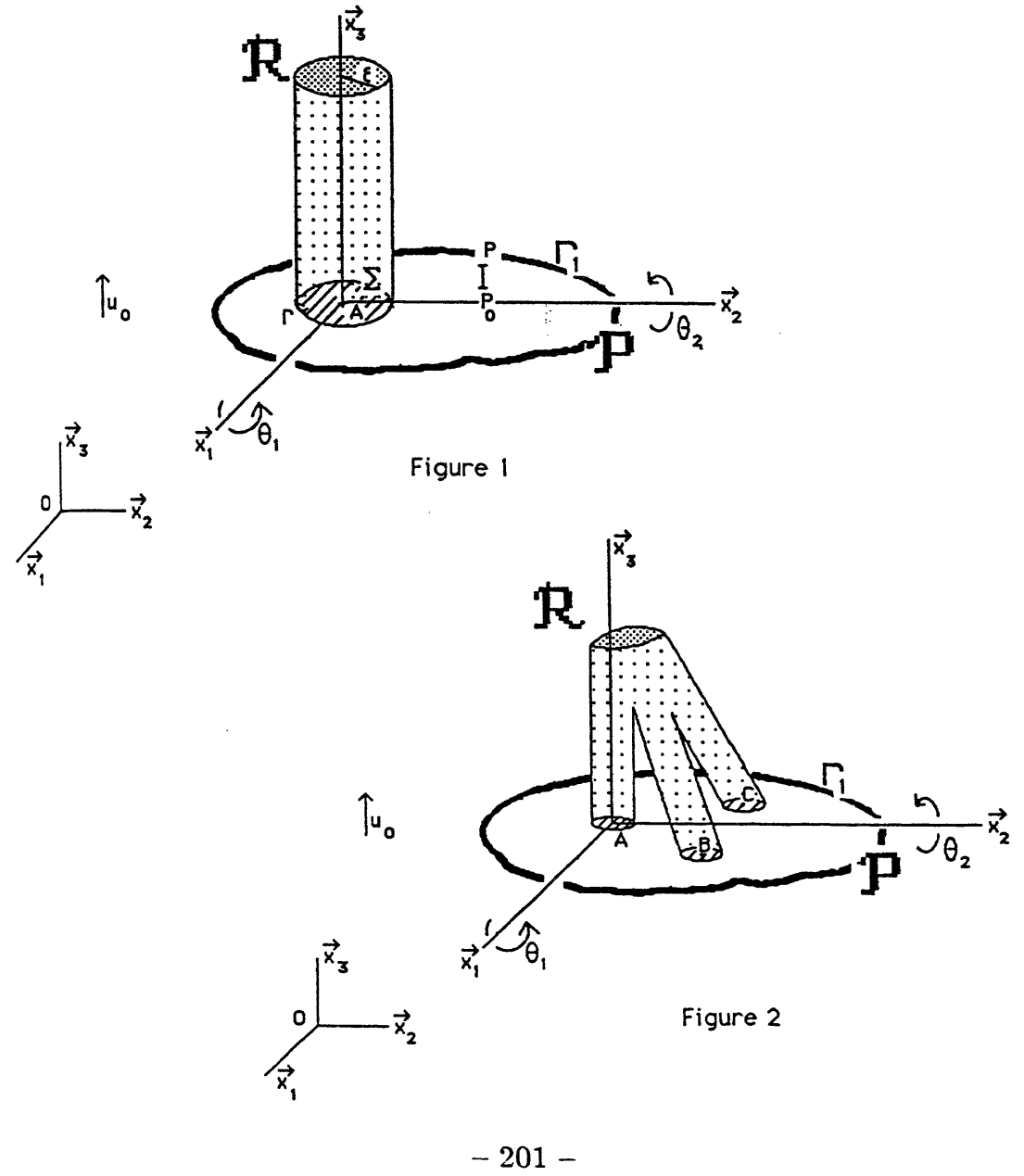




\section{A. Campbell}

\section{II.2. - Théorèmes d'existence et d'unicité}

On cherche une solution en déplacement du problème

$$
P \in \mathcal{P} \quad \overrightarrow{P_{0} P}=W\left(x_{1}, x_{2}\right) e^{i \omega t} \vec{x}_{3}
$$

où $P$ est de coordonnées $\left(x_{1}, x_{2}\right)$. Les conditions aux limites sont :

$$
\forall P \in \Sigma \quad W\left(x_{1}, x_{2}\right)=U_{0}-\Theta_{2} x_{1}+\Theta_{1} x_{2},
$$

le contour $\Gamma_{1}$ étant libre d'effort.

L'équation de vibration des plaques en flexion est ici :

$$
\Delta(\Delta W)-\frac{\rho h \omega^{2}}{N} W=0 .
$$

On obtient alors la formulation variationnelle suivante [cf.4] : Dans l'ensemble admissible,

$$
\mathcal{W}_{a d}=\left\{V \in H^{2}(\mathcal{P}) / V=U_{0}-\Theta_{2} x_{1}+\Theta_{1} x_{2} \text { sur } \Sigma\right\}
$$

on cherche $W$ tel que, pour tout $V$ appartenant à $\mathcal{W}_{a d}$,

$$
a(W, V-W)=c^{4} \int_{\mathcal{P}} W(V-W) d s
$$

où on a posé

$$
\begin{aligned}
a(W, V)= & \int_{\mathcal{P}}\left[\frac{\partial^{2} W}{\partial x_{i}^{2}} \frac{\partial^{2} V}{\partial x_{i}^{2}}+v\left(\frac{\partial^{2} W}{\partial x_{1}^{2}} \frac{\partial^{2} V}{\partial x_{2}^{2}}+\frac{\partial^{2} W}{\partial x_{2}^{2}} \cdot \frac{\partial^{2} V}{\partial x_{1}^{2}}\right)\right. \\
& \left.+2(1-v) \frac{\partial^{2} V}{\partial x_{1} \partial x_{2}} \frac{\partial^{2} W}{\partial x_{1} \partial x_{2}}\right] N d s
\end{aligned}
$$

et $c^{4}=\rho h \omega^{2}$.

$N$ est le coefficient de rigidité de flexion de $\mathcal{P}$ :

$$
N=\frac{E h^{3}}{12\left(1-v^{2}\right)} \text {. }
$$

On notera aussi :

$$
\mathcal{A}(W, V)=a(W, V)-c^{4} \int_{\mathcal{P}} W \cdot V d s .
$$


THÉORÈME 1. - Soit l'espace

$$
\mathcal{W}_{a d_{0}}(\Sigma)=\left\{V \in H^{2}(\mathcal{P}) / V=0 \text { sur } \Sigma\right\}
$$

$\mathcal{W}_{a d_{0}}(\Sigma)$ est un espace de Hilbert. La forme bilinéaire a est coercive sur $\mathcal{W}_{a d_{0}}(\Sigma)$ si $\Sigma$ est de mesure non nulle. $a^{1 / 2}$ défnit alors sur $\mathcal{W}_{a d_{0}}(\Sigma)$ une norme équivalente à la norme $H^{2}$.

La démonstration est çassique :

Sur $H^{2}(\mathcal{P})$ la norme $H^{2}$ est équivalente à

$$
\left[\|\cdot\|_{H^{1}}^{2}+a(., .)\right]^{1 / 2}
$$

[cf.5]. $a$ est une forme bilinéaire continue sur $H^{2}(\mathcal{P})$. Donc il faut montrer ici que

$$
\forall V \in \mathcal{W}_{a d_{0}}(\Sigma) \quad a(V, V) \geq C\|V\|_{H^{1}}^{2}
$$

ou encore qu'il existe une constante positive $C$ telle que

$$
\forall V \in \mathcal{W}_{a d_{0}}(\Sigma) \text { tel que }\|V\|_{H^{1}}=1, \quad a(V, V) \geq C>0 .
$$

Si cela n'était pass vrai il existerait une suite $\left(V_{n}\right)$ dans $\mathcal{W}_{a d_{0}}(\Sigma)$ telle que

$$
\left\|V_{n}\right\|_{H^{1}}=1 \text { et } a\left(V_{n}, V_{n}\right) \leq \dot{1 / n}
$$

donc d'après (8), la suite serait bornée dans $H^{2}$ donc fortement convergente à une extraction près dans $H^{1}$ vers une limite $V_{0}$.

D'après la continuité de $a$ on aurait donc

$$
a\left(V_{0}, V_{0}\right)=0 \text { et }\left\|V_{o}\right\|_{H_{1}}=1
$$

$V_{0}$ serait donc un déplacement rigide dans $\mathcal{W}_{a d_{0}}(\Sigma) . \Sigma$ étant de mesure non nulle, $V_{0}$ doit être nul ce qui est absurde. Notons qu'il suffit que $V_{0}$ s'annule en trois poits non alignés pour assurer la même conclusion.

Il existe donc $\alpha(\Sigma)$ strictement positif tel que

$$
\forall V \in \mathcal{W}_{a d_{0}}(\Sigma) a(V, V) \geq \alpha(\Sigma)\|V\|_{H^{2}(\mathcal{P})}^{2}
$$

$a$ étant continue, $a^{1 / 2}$ est une norme équivalente à la norme $H^{2}$.

THÉORÈME 2. $-I l$ existe une suite croissante $\left(\lambda_{n}(\Sigma)\right)_{n \geq 0}$ de nombres positifs tels que le problème, 
A. Campbell

$$
\begin{gathered}
\text { "trouver } W \text { appartenant à } \mathcal{W}_{a d_{0}}(\Sigma) \text { tel que } \\
\forall V \in \mathcal{W}_{a d_{0}}(\Sigma) a(V, W)=\lambda_{n}(\Sigma) \int_{\mathcal{P}} V . W d s^{\prime},
\end{gathered}
$$

admette des solutions non nulles.

De plus on $a$ :

$$
\lambda_{n}(\Sigma)=\inf _{\substack{V \in \mathcal{W}_{\text {ado }}(\Sigma \neq 0) \\ V \neq 0}} \frac{a(V, V)}{\int_{\mathcal{P}} V^{2} d s}
$$

Démonstration. - On introduit l'opérateur $\mathcal{F}$ défini par

$$
\forall V, W \in \mathcal{W}_{a d_{0}}(\Sigma) \quad a(V, \mathcal{F}(W))=\int_{\mathcal{P}} V . W d s
$$

$\mathcal{F}$ est un opérateur auto-adjoint compact et son spectre est constitué de valeurs propres isolées notées $\left(1 / \lambda_{n}\right)$ [cf.6]. Par quotient de Rayleigh on a donc (11). Les $\lambda_{n}$ correspondent aux vibrations propres de la plaque encastrée suivant $\Sigma$.

TIIÉORÈmE 3. - Soit $L$ une forme linéaire continue sur $H^{2}(\mathcal{P})$. Si c $c^{4}$ est différent de $\lambda^{n}(\Sigma)$ pour tout $n$, alors le problème défini par :

$$
\begin{aligned}
& \text { "Déterminer } W(\Sigma) \in \mathcal{W}_{a d_{0}}(\Sigma) \text { et tel que } \\
& \forall V \in \mathcal{W}_{a d_{0}}(\Sigma) \mathcal{A}(W(\Sigma), V)=L(V) "
\end{aligned}
$$

admet une solution unique.

$\mathcal{A}$ est la forme bilinéaire définie en (7).

$\mathcal{A}$ est non coercive mais seulement faiblement coercive sur $\mathcal{W}_{a d_{0}}(\Sigma)$ c'està-dire qu'il existe $\gamma(\Sigma)$ constante positive telle que :

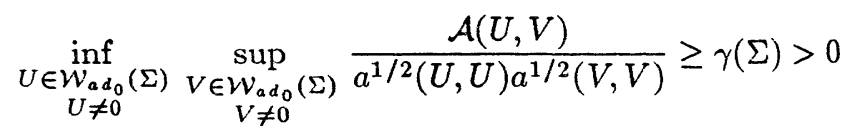

[cf.7].

L'opérateur compact $\mathcal{F}$ est de norme $\frac{1}{\lambda_{0}(\Sigma)}$. On peut alors choisir [cf. 8 ]

$$
\gamma(\Sigma)=\frac{1}{1+\frac{c^{4}}{\lambda_{o}(\Sigma)}} \times \inf _{n \in N}\left|1-\frac{c^{4}}{\lambda_{n}(\Sigma)}\right|
$$




\section{Impédance d'une plaque élastique}

qui est bien strictement positive si $c^{4}$ est différent de tous les $\lambda_{n}(\Sigma)$.

Les problèmes qui se poseront dans la deuxième partie seront liés au comportement de $\alpha(\Sigma)$ défini en $(10)$, des $\lambda_{n}(\Sigma)$ et de la constante $\gamma(\Sigma)$ quand la mesure de $\Sigma$ tend vers zéro.

THÉORÈME 4. - Si $c^{4}$ est différent de $\lambda_{n}(\Sigma)$ pour tout $n$, le problème variationnel défini en (4) et (5) admet une solution unique.

En effet, pour tout élément $V$ de l'ensemble $\mathcal{W}_{a d}$ défini en (4), on pose :

$$
V=U_{0}-\Theta_{2} x_{1}+\Theta_{1} x_{2}+V^{0}
$$

Comme $\mathcal{P}$ est bornée, $U_{0}-\Theta_{2} x_{1}+\Theta_{1} x_{2}$ est dans $H^{2}(\mathcal{P})$ et donc $V^{0}$ est aussi dans $H^{2}(\mathcal{P})$ et de trace nulle sur $\Sigma$. De plus, la forme bilinéaire $a$ ne faisant intervenir que des dérivées secondes, on a

$$
a(W, V-W)=a\left(W^{0}, V^{0}-W^{0}\right)
$$

et donc

$$
\mathcal{A}(W, V-W)=\mathcal{A}\left(W^{0}, V^{0}-W^{0}\right)+L\left(V^{0}-W^{0}\right)
$$

où on a posé

$$
L(V)=c^{4} \int_{\mathcal{P}}\left(U_{0}-\Theta_{2} x_{1}+\Theta_{1} x_{2}\right) V d s
$$

Le problème est alors équivalent à la détermination de $W^{0}$ dans $\mathcal{W}_{a d_{0}}(\Sigma)$ tel que

$$
\forall V^{0} \in \mathcal{W}_{a d_{0}}(\Sigma) \quad \mathcal{A}\left(W^{0}, V^{0}\right)=L\left(V^{0}\right)
$$

Il suffit alors d'appliquer le théorème 3 .

THÉORÈME 5. - La solution générale du théorème 4 peut s'écrire :

$$
U_{0} W_{0}+\Theta_{1} W_{1}+\Theta_{2} W_{2}
$$

où $W_{0}$ est la solution unique obtenue avec le second membre

$$
L_{0}=c^{4} \int_{\mathcal{P}} V d s
$$

$W_{1}$ avec le second membre

$$
\begin{aligned}
L_{1}= & c^{4} \int_{\mathcal{P}} x_{2} V d s \\
& -205-
\end{aligned}
$$




\section{A. Campbell}

et $W_{2}$ avec

$$
L^{2}=-c^{4} \int_{\mathcal{P}} x_{1} V d s
$$

\section{II.3. - Calcul de la matrice d'impédance}

TIÉORÈME 6.- La matrice d'impédance (1) peut se mettre sous la forme suivante si $c^{4}$ est différent de $\lambda_{n}(\Sigma)$ :

$$
\mathcal{I}=\rho h \omega^{2}\left[\begin{array}{ccc}
m & m x_{2}(G) & -m x_{1}(G) \\
m x_{2}(G) & A_{22} & A_{12} \\
-m ! x_{1}(G) & A_{12} & A_{11}
\end{array}\right]+\left[\mathcal{A}\left(W_{i}, W_{j}\right)\right]_{i, j=0,1,2}
$$

où on a posé

$$
\begin{gathered}
m=\int_{\mathcal{P}-\Sigma} d s, \quad m x_{i}(G)=\int_{\mathcal{P}-\Sigma} x_{i} d s \\
A_{\alpha, \beta}=(-1)^{|\beta-\alpha|} \int_{\mathcal{P}-\Sigma} x_{\alpha} x_{\beta} d s
\end{gathered}
$$

$\mathcal{A}$ est la forme bilinéaire (7) et les $W_{i}$ sont les solutions du théorème 5.

La première partie de cette expression matricielle est l'impédance du "solide indéformable" $\mathcal{P}-\Sigma$. La seconde matrice traduit le caractère élastique. Démontrons le théorème :

On suppose que

$$
U_{0}=1 \quad \Theta_{1}=\Theta_{2}=0
$$

ce qui va nous fournir la première colonne de la matrice d'impédance. Les deux autres sont obtenues de manière analogue. Soit $V$ un élément quelconque de $H^{2}(\mathcal{P})$ et $W_{0}$ la solution définie au théorème (5); d'après le théorème de Green on peut écrire :

$$
a\left(W_{0}, V\right)-\int_{\Gamma} F . V d \gamma+\int_{\Gamma} M_{\tau} \frac{\partial V}{\partial n} d \gamma=\rho h \omega^{2} \int_{\mathcal{P}-\Sigma}\left(1+W_{0}\right) V d s
$$

où $\Gamma$ est le contour de $\Sigma$.

$F$ et $M_{\tau}$ désignent les densités de forces et de moments exercées sur $\Gamma$ (cf.[4]). C'est en réduisant en $A$ ces actions qu'on exhibe les termes d'impédance. 
Pour calculer $\int_{\Gamma} F d \gamma$ et $\int_{\Gamma} M I_{\tau} d \gamma$, il suffit alors de prendre successivement $V=1, V=x_{2}$ et $V=x_{1}$. On obtient :

$$
\begin{aligned}
I_{00} & =-\int_{\Gamma} F d \gamma=\rho h \omega^{2} \int_{\mathcal{P}-\Sigma}\left(1+W_{0}\right) d s \\
& =\rho h \omega^{2} m+\mathcal{A}\left(W_{0}, W_{0}\right)
\end{aligned}
$$

et

$$
\begin{aligned}
I_{10} & =\rho h \omega^{2} \int_{\mathcal{P}-\Sigma}\left(1+W_{0}\right) x_{2} d s \\
& =\rho h \omega^{2} m x_{2}(G)+\mathcal{A}\left(W_{0}, W_{1}\right)
\end{aligned}
$$

puis

$$
I_{20}=-\rho h \omega^{2} m x_{1}(G)+\mathcal{A}\left(W_{0}, W_{2}\right) .
$$

On procède de même pour les autres termes $I_{i j}$.

Remarquons que la forme obtenue pour $\mathcal{I}$ montre que cette matrice est SYMETRIQUE.

\section{Deuxième partie}

\section{Liaison en trois points; limite de la matrice d'impédance}

\section{III.1.-Cas où la soudure $\Sigma$ se réduit à 3 points $\mathrm{A}, \mathrm{B}, \mathrm{C}$}

Soient trois points $A, B$ et $C$ non alignés où la soudure $\Sigma$ est réalisée. Certains résultats analogues à ceux de la première partie sont valables; on utilisera les notations suivantes :

$$
\overline{\mathcal{W}}_{a d_{0}}=\left\{V \in H^{2}(\mathcal{P}) / V(A)=V(B)=V(C)=0\right\}
$$

$a^{1 / 2}$ est une norme sur l'espace de Hilbert $\overline{\mathcal{W}}_{a d_{0}}$ et cette norme est équivalente à la norme $H^{2}$. La démonstration est celle du théorème 1 .

La suite $\left(\lambda_{n}(\Sigma)\right)$ du théorème 2 sera notée ici $\overline{\left(\lambda_{n}\right)}$. Le théorème 3 est toujours valable et $\bar{\gamma}$ sera la constante de coercivité faible définie en (14). Pour $c^{4}$ différent de $\overline{\lambda_{n}}$ pour tout $n$, on définit donc les solutions $\bar{W}_{0}, \bar{W}_{1}$ et $\bar{W}_{2}$ comme au théorème 5 , et plus généralement $\bar{W}$.

Cependant il n'est plus possible de calculer la matrice d'impédance en utilisant le théorème de Green comme en (18), car les termes de contour 


\section{A. Campbell}

sur $\Gamma$ disparaissent. On va donc introduire un $\Sigma(\epsilon)$ de mesure non nulle constitué de trois disques $d(A, \epsilon), d(B, \epsilon)$ et $d(C, \epsilon)$ de centres $A, B$ et $C$ et de rayon $\epsilon$ (cf Figure 2).

On a donc défini la soudure $\Sigma$ par :

$$
\Sigma(\epsilon)=d(A, \epsilon) \cup d(B, \epsilon) \cup d(C, \epsilon) .
$$

On utilise ici les notations $\mathcal{W}_{a d_{0}}(\epsilon)$ pour $\mathcal{W}_{a d_{0}}(\Sigma), \lambda_{n}(\epsilon)$ pour $\lambda_{n}(\Sigma), \gamma(\epsilon)$ pour $\gamma(\Sigma)$ et on notera $W_{i}(\epsilon) i=1,2,3$, les solutions du théorème (5). On peut alors calculer la matrice d'impédance comme au théorème 6 . On va s'intéresser alors au comportement quand $\epsilon$ tend vers zéro des termes du type

$$
\mathcal{A}\left(W_{i}(\epsilon), W_{j}(\epsilon)\right)
$$

\section{III.2. - Passage à la limite quand $\epsilon$ tend vers zéro}

On a évidemment :

$$
\overline{\mathcal{W}}_{a d_{0}} \subset \mathcal{W}_{a d_{0}}(\epsilon)
$$

et donc

$$
\bar{\lambda}_{0}=\inf _{\substack{V \in \overline{\mathcal{W}}_{\text {ado }} \\ V \neq 0}} \frac{a(V, V)}{\int_{\mathcal{P}} V^{2} d s} \leq \inf _{\substack{V \in \mathcal{W}_{\text {ad }}(\epsilon) \\ V \neq 0}} \frac{a(V, V)}{\int_{\mathcal{P}} V^{2} d s}=\lambda_{0}(\epsilon) \leq \lambda_{n}(\epsilon) .
$$

Les fonctions $\lambda_{n}(\epsilon)$, décroissantes quand $\epsilon$ tend vers zéro sont done minorées par $\bar{\lambda}_{0}$. Elles admettent une limite :

$$
\lambda_{n}^{*}=\lim _{\epsilon \rightarrow 0} \lambda_{n}(\epsilon)
$$

D'autre part si dans (13) on remplace $U$ par $W(\epsilon)$ solution du problème (12) on obtient :

$$
\frac{1}{a^{1 / 2}(W(\epsilon), W(\epsilon))} \sup _{\substack{V \in \mathcal{W}_{a d_{0}}(\epsilon) \\ V \neq 0}} \frac{L(V)}{a^{1 / 2}(V, V)} \geq \gamma(\epsilon) .
$$

Dans $\overline{\mathcal{W}}_{a d_{0}}, a^{1 / 2}$ est une norme équivalente à la norme $H^{2}$ donc :

$$
\begin{gathered}
\|W(\epsilon)\|_{H^{2}} \leq \frac{K}{\gamma(\epsilon)} \\
-208-
\end{gathered}
$$




\section{Impédance d'une plaque élastique}

où $K$ est indépendant de $\epsilon$, car la norme de $L$ dans $\mathcal{W}_{a d_{0}}(\epsilon)$ est majorée par la norme dans $\overline{\mathcal{W}}_{a d_{0}}$. L'existence de $W(\epsilon)$ et son unicité sont réalisées si $c^{4}$ est différent de $\lambda_{n}(\epsilon)$ pour $\epsilon$ suffisamment petit. On supposera ici

$$
\forall n \quad c^{4} \neq \lambda_{n}^{*} \quad \text { et } \quad c^{4} \neq \bar{\lambda}_{n} .
$$

La constante $\gamma(\epsilon)$ définie en (14) admet alors une limite non nulle et $W(\epsilon)$ est donc borné dans $H^{2}(\mathcal{P})$. $W(\epsilon)$ admet donc une limite faible que nous noterons $S$.

Remarquons que $W(\epsilon)$ tend vers $S$ fortement dans $H^{3 / 2}(\mathcal{P})$ et que donc $S$ est continue sur $\mathcal{P}$. On a donc

$$
S(A)=S(B)=S(C)=0
$$

et donc

$$
S \in \overline{\mathcal{W}}_{a d_{0}}
$$

Il reste à montrer que $S$ est confondue avec $\bar{W}$. Pour cela on a besoin d'un théorème de densité :

THÉORÈME 7. - Soit

$$
\overline{\mathcal{W}}_{a d_{0}}=\left\{V \in H^{2}(\mathcal{P}) / V(A)=V(B)=V(C)=0\right\}
$$

Alors l'ensemble

$$
\left\{V \in H^{2}(\mathcal{P}) / \exists \delta>0 \text { tel que } V=0 \text { sur } \Sigma(\delta)\right\}
$$

est dense dans $\overline{\mathcal{W}}_{a d_{0}}$.

qui résulte immédiatement du théorème 8 :

THÉORÈme 8. - Soit

$$
\widehat{\mathcal{W}}_{a d_{0}}=\left\{V \in H^{2}(\mathcal{P}) / V(A)=0\right\} \text {. }
$$

Alors $\mathcal{W}^{*}=\left\{V \in H^{2}(\mathcal{P}) / \exists \delta>0\right.$ tel que $V=0$ sur un disque de centre 0 et de rayon $\delta\}$ est dense dans $\widehat{\mathcal{W}}_{a d_{0}}$.

Ce théorème est une généralisation d'une propriété de densité des fonctions contantes au voisinage d'un point dans $H^{1}(\mathcal{P})$ [cf.9 et 10]. Pour démontrer le théorème 8 , on va montrer que toute fonction $\theta$ de l'ensemble

$$
\mathcal{D}_{0}(\overline{\mathcal{P}})\{\theta \in \mathcal{D}(\overline{\mathcal{P}}) \text { tel que } \theta(A)=0\}
$$




\section{A. Campbell}

peut être approchée par une fonction $\theta_{R}$ de $H^{2}(\mathcal{P})$ nulle sur un disque de centre $A$ et de rayon $R / 2$.

Le théorème 8 en résultera par densité de $\mathcal{D}_{0}(\overline{\mathcal{P}})$ dans $\widehat{\mathcal{W}}_{a d_{0}}$ qui résulte de la densité de $\mathcal{D}(\overline{\mathcal{P}})$ dans $H^{2}(\mathcal{P})$. [cf.11].

$\theta$ étant donné, on construit $\theta_{R}$ de la façon suivante $:(r, \varphi)$ désignant les coordonnées polaires dans $A x_{1} x_{2}$ :

$$
\begin{array}{lll}
\theta_{R}=0 & \text { si } & r<\frac{R}{2} \\
\theta_{R}=\theta & \text { si } & r>R
\end{array}
$$

et, si $r$ est compris entre $R / 2$ et $R$,

$$
\begin{aligned}
\theta_{R}(r, \varphi) & =\frac{(r-R / 2)^{2}}{(R / 2)^{2}}\left\{\left(\frac{\partial \theta}{\partial r}(R, \varphi)-\frac{4}{R} \theta(R, \varphi)\right) r\right. \\
& \left.+5 \theta(R, \varphi)-R \frac{\partial \theta}{\partial r}(R, \varphi)\right\} .
\end{aligned}
$$

Il est facile de voir que $\theta_{R}$ est de classe $C^{1}$ sur $\mathcal{P}$, donc que $\theta_{R}$ est dans $H^{2}(\mathcal{P})$. D'autre part, des calculs très simples montrent que la norme du vecteur $\left(\frac{\partial^{2} \theta_{R}}{\partial r^{2}}, \frac{1}{r} \frac{\partial^{2} \theta_{R}}{\partial r \partial \varphi}, \frac{1}{r^{2}} \frac{\partial^{2} \theta_{R}}{\partial \varphi^{2}}\right)$ est majorée par $\frac{K}{R}$ quand $R$ tend vers zéro, $K$ étant une constante positive.

La mesure du support de la fonction $\theta-\theta_{R}$ est $\pi R^{2}$ et donc $\theta-\theta_{R}$ est bornée dans $H^{2}(\mathcal{P}) \theta-\theta_{R}$ tend donc faiblement vers zéro dans $H^{2}(\mathcal{P})$.

On a montré que pour tout $V$ de $\widehat{\mathcal{W}}_{a d_{0}}$, il existe une suite $\left(V_{i}\right)$ de $\mathcal{W}^{*}$ convergeant faiblement vers $V$ dans $H^{2}(\mathcal{P})$. $\widehat{\mathcal{W}}_{a d_{0}}$ :

Soit $U$ de $\widehat{\mathcal{W}}_{a d_{0}}$ choisi dans l'espace orthogonal à l'adhérence de $\mathcal{W}^{*}$ dans

On peut trouver $U_{i}$ dans $\mathcal{W}^{*}$ convergeant vers $U$ faiblement dans $H^{2}(\mathcal{P})$ et on a

$$
\lim \left(U, U_{i}\right)_{H^{2}(\mathcal{P})}=0 \quad \operatorname{car} U \in\left(\overline{\mathcal{W}}^{*}\right)^{\perp}
$$

et

$$
\lim \left(U, U_{i}\right)_{H^{2}(\mathcal{P})}=\|U\|_{H^{2}}
$$

par convergence faible. $U$ est donc nul est $\widehat{\mathcal{W}}_{a d_{0}}$ est bien la fermeture de $\mathcal{W}^{*}$, ce qui achève la démonstration du théorème.

Il reste alors à montrer que $S$ et $\bar{W}$ sont confondus. 
Soit deux nombres positifs $\epsilon$ et $\delta,(0<\epsilon<\delta)$, on a :

$$
\mathcal{W}_{a d_{0}}(\delta) \subset \mathcal{W}_{a d_{0}}(\epsilon)
$$

Donc

$$
\forall \epsilon<\delta, \forall V(\delta) \in \mathcal{W}_{a d_{0}}(\delta), \mathcal{A}(W(\epsilon), V(\delta))=L(V(\delta))
$$

En faisant tendre $\epsilon$ vers zéro, il vient :

$$
\mathcal{A}(S, V(\delta))=L(V(\delta))
$$

et par densité (théorème 7)

$$
\forall \bar{V} \in \overline{\mathcal{W}}_{a d_{0}} \quad \mathcal{A}(S, \bar{V})=L(\bar{V})
$$

La solution $\bar{W}$ étant unique on a donc bien : $S=\bar{W}$.

Les termes $\mathcal{A}\left(W_{i}(\epsilon), W_{j}(\epsilon)\right)$ obtenus dans la matrice d'impédance tendent donc vers $\mathcal{A}\left(\bar{W}_{i}, \bar{W}_{j}\right)$. En effet

$$
\begin{aligned}
\mathcal{A}\left(W_{i}(\epsilon), W_{j}(\epsilon)\right) & =L_{j}\left(W_{i}(\epsilon)\right) \\
& =\mathcal{A}\left(W_{i}(\epsilon), W_{j}(\eta)\right)
\end{aligned}
$$

ceci pour tout nombre $\eta$ inférieur à $\epsilon$. Donc par densité

$$
\mathcal{A}\left(W_{i}(\epsilon), W_{j}(\epsilon)\right)=\mathcal{A}\left(W_{i}(\epsilon), \bar{W}_{j}\right)
$$

et

$$
\lim _{\epsilon \rightarrow 0} \mathcal{A}\left(W_{i}(\epsilon), \bar{W}_{j}\right)=\mathcal{A}\left(\bar{W}_{i}, \bar{W}_{j}\right)
$$

On obtient donc dans ce cas une matrice d'impédance limite qui a la forme (17) en utilisant les solutions $\bar{W}_{i}$.

Pour obtenir ce résultat nous avons supposé en (21) que $c^{4}$ est différent de tous les nombres $\lambda_{n}^{*}$ et $\bar{\lambda}_{n}$. En fait ces deux suites sont confondues; en effet par le théorème 8 et en utilisant le quotient de Rayleigh, on obtient facilement l'égalité de $\lambda_{0}^{*}$ et $\bar{\lambda}_{0}$. Pour les valeurs propres d'ordre supérieur, on peut par exemple utiliser le principe de "Min-Max" [cf.12]. 


\section{A. Campbell}

\section{Conclusions}

Considérons une plaque élastique reliée à un support rigide suivant un ensemble $\Sigma$.

Si la mesure de $\Sigma$ est strictement positive, alors les propriétés d'existence et d'unicité des déplacements permettent le calcul de la matrice d'impédance en utilisant les solutions de trois problèmes de base. On a montré ici de plus que si la mesure de $\Sigma$ est nulle mais si l'existence et l'unicité des solutions sont satisfaites, par exemple si $\Sigma$ est constituée de trois points non alignés, alors on peut encore définir trois solutions de base, la notion de matrice d'impédance s'obtient alors par passage à la limite : la limite de la matrice d'impédance a la forme obtenue précédemment mais en utilisant les solutions limites.

Il reste à étudier le cas intermédiaire : quel est par exemple le comportement de la matrice d'impédance si $\Sigma$ se réduit à un seul point. Des résultats particuliers dans le cas de plaques simples comme les disques, montrent l'évanouissement de certains termes d'impédance mais la résolution du cas général constitue un problème ouvert.

\section{Références}

[1] Crellin (E.), Janssens (F.).- Derivation and Combination of impedance matrices for flexible satellites, Rapport E.S.A., ${ }^{198} \mathbf{4}$.

[2] Kelkel (K.), HAGEDORN (P.). - Study of plate impedance, Rapport E.S.A., avril 1985 .

[3] CAMPBELl (A.). - Impédance d'un solide tridimensionnel relié à un support rigide vibrant., C.R.A.S., t. 304, série II, n. ${ }^{\circ} 13,1987$.

[4] Duvaut (G.), Lions (J.L.).- Inéquations en mécanique et en physique, Dunod, 1972.

[5] ReKtorys (K.).- Variational methods in mathematical, science and engineerling-Reidel publishing company, 1980.

[3] Riesz (F.), NAGY (B.SZ.). - Leçons d'analyse fonctionnelle, Gauthier-Villars, 1955.

[7] Brezzi (F.).- On the existence, uniqueness and approximation of saddle-point problems arising from Lagrangian multipliers, Rev. Fr. Automati. Inform. Recherche Opérat. Numer. Anal., t. R2, 1974, p. 129-151.

[8] Brezis (H.).- Analyse fonctionnelle, Masson, $19^{86 .}$

[9] Caillerie (D.).-Etude de quelques problèmes de perturbations en théorie de l'élasticité et de la conduction thermique-Thèse d'Etat. Université de Paris VI. 1982 . 
Impédance d'une plaque élastique

[10] Pham Huy (H.), Sanchez Palencia (E.). - Phénomènes de transmission à travers des couches limites de conductivité élevée, J. Math. Anal. and Appl., t. 47, $\mathrm{n}^{\circ} 2,1974$.

[11] Lions (J.L.), Magenes (E.), Problèmes aux limites non homogènes. - Dunod, 1968.

[12] Courant (R.), Hilbert (D.). - Méthods of mathematical physics, Interscience, 1937.

(Manuscrit reçu le 5 septembre 1988) 